\title{
Nucleation and Growth Behavior of CdSe Nanocrystals Synthesized in the Presence of Oleylamine Coordinating Ligand
}

\author{
X. Huang, ${ }^{\circledR}$ V. K. Parashar, ${ }^{\circledR}$ and M. A. M. Gijs* \\ Laboratory of Microsystems, Ecole Polytechnique Fédérale de Lausanne (EPFL), CH-1015 Lausanne, Switzerland \\ Supporting Information
}

ABSTRACT: We established the dual role played by oleylamine $(\mathrm{OAm})$ during the synthesis of CdSe nanocrystals (NCs) by the hot injection method. Earlier reports suggest its role either as nucleating or as passivating agent in controlling the growth of CdSe NCs. Remarkably, by exploring four different synthesis routes, in which the reactant addition, timing, and concentration were varied, we found that both these two phenomena coexist and control synthesis. While examining if there is any effect of concentration of OAm on

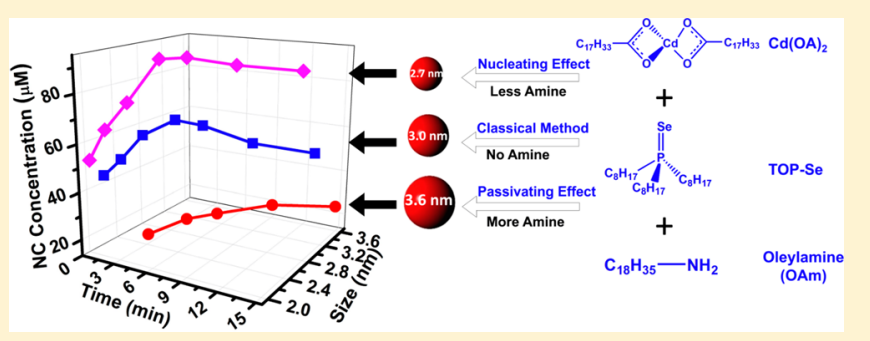
this synthesis, we found that at lower contents of OAm $(<0.5 \mathrm{mmol})$ it prominently acts as an agent acting on nucleation, increasing the number of nuclei, reducing the nuclei (initial monomer units) size, and thereby increasing the NCs concentration resulting in a small NCs size, down to $2.7 \mathrm{~nm}$. Whereas at higher contents $(>1.0 \mathrm{mmol})$, it served more as a passivating agent by deterring both nucleation and growth processes, so generating fewer NCs with larger size up to $3.6 \mathrm{~nm}$. Thus, adjusting the influence between nucleation and passivation, we can better control the final NCs size and so tune their size-dependent optical properties.

\section{INTRODUCTION}

Cadmium selenide NCs (CdSe NCs), in recent years, emerged in a wide range of electrical and optical applications. ${ }^{1,2}$ Synthesis via the hot injection technique led to formation of NCs having better luminescent performance, ${ }^{3-5}$ which was further augmented with the exploration and understanding of a diversity of surface ligands to effectively control size, shape, crystal structure, and luminescent performance of the NCs. ${ }^{6-9}$ One class of surface ligand of special interest are alkylamines, which weakly bond to the surface of NCs, forming a high packing density, effectively eliminating the unsatisfied dangling bonds, tuning the NCs size and size distribution, and therefore resulting in an adjustment of the ultraviolet-visible (UV-vis) absorbance, photoluminescence (PL), and an enhancement of the photoluminescence quantum yield (PLQY). ${ }^{10-14}$ However, the role of the amine, particularly oleylamine (OAm), commonly used owing to the same structural units as found in the oleate group of cadmium oleate, is not understood to the extent that contradictory explanations for both NCs nucleation and passivation stages were reported. Few authors have reported that its addition would activate the cadmium precursor and thus promote the nucleation of NCs. ${ }^{15-17}$ These results were based upon the evidence that a smaller nuclei size can be observed at an earlier time and higher nanocrystal concentration as well as growth rate were found when primary amines were used, whereas others have reasoned a delayed nucleation in the amine-containing reaction, which was proposed to result from formation of low-activity $\mathrm{Cd}$-amine complexes by the coordination strength or steric hindrance effect. ${ }^{18,19}$ Recently, it was demonstrated that alkylamines restrained monomer activity by inhibiting the cleavage of the $\mathrm{P}=\mathrm{Se}$ bond in the $\mathrm{Se}$ precursor while increasing the growth rate of $\mathrm{CdSe} \mathrm{NCs}$ and reducing the NCs concentration. ${ }^{12,20}$ These contradictory research results make it obvious that more in-depth studies are necessary to establish the role of OAm in NCs synthesis.

\section{EXPERIMENTAL SECTION}

Materials. Commercially available chemicals, cadmium oxide (CdO, 99.5\% purity), 1-octadecene (ODE), oleic acid (OA), oleylamine (>98\% purity, OAm), trioctylphosphine (TOP, 97\% purity), selenium powder (Se), hexane, chloroform, and ethanol were purchased from Sigma-Aldrich.

Synthesis of the CdSe NCs. To minimize the influence of air and water, we used the Schlenk line to create an inert environment for the reaction. Before the synthesis of the CdSe NCs, the precursors were prepared in advance. A clear cadmium oleate $\left(\mathrm{Cd}(\mathrm{OA})_{2}\right)$ precursor solution $(0.1 \mathrm{M})$ was obtained by dissolving $3 \mathrm{mmol}$ of $\mathrm{CdO}$ in 12 $\mathrm{mmol}$ of $\mathrm{OA}$ at $200{ }^{\circ} \mathrm{C}$ for more than $6 \mathrm{~h}$ under the protection of argon atmosphere, followed by diluting in $25.8 \mathrm{~mL}$ of degassed ODE. For TOP-Se precursor, TOP solution and Se powder in a molar ratio of 1:1 were mixed and stirred at room temperature overnight in a glovebox and then diluted in ODE to acquire a concentration of $0.5 \mathrm{M}$. The OAm should also be degassed and heated to $50{ }^{\circ} \mathrm{C}$ to get fully clear solution just before addition.

The synthesis of CdSe NCs was performed based on classical hot injection methods with a modification. As discussed in Figure 1 (see article), four routes were designed to study the influence of amine on CdSe NCs. In route $1,0.5 \mathrm{~mL}$ of $\mathrm{Cd}$ precursor was mixed with $4 \mathrm{~mL}$

Received: April 18, 2017

Revised: February 23, 2018

Published: May 10, 2018 


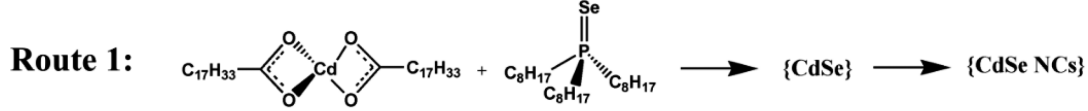

Route 2: $\quad \mathrm{C}_{17} \mathrm{H}_{33}-\mathrm{C}_{\mathrm{N}}$

Route 3:

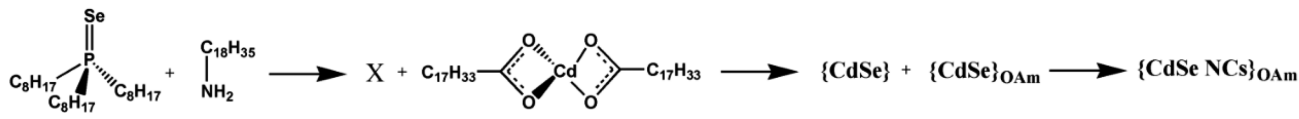

Route 4:

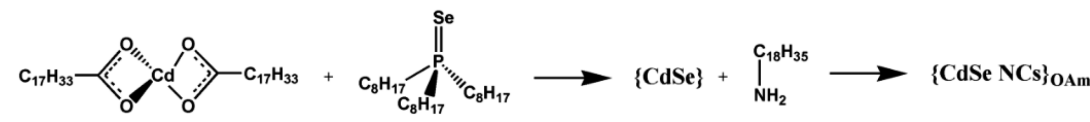

Figure 1. Schematic of designed four reaction routes to investigate the influence of oleylamine (OAm) on the synthesis of CdSe NCs at a reaction temperature of $210^{\circ} \mathrm{C}$. Route 1: Cd oleate precursor $\left(\mathrm{Cd}(\mathrm{OA})_{2}\right)$ in octadecene (ODE) solution is prepared, and then the Se precursor (TOP-Se) is injected. Route 2: various concentrations of OAm are added to the $\mathrm{Cd}(\mathrm{OA})_{2}$ in ODE solution, after which TOP-Se is injected. Route 3: various concentrations of OAm and TOP-Se are mixed together in ODE, after which $\mathrm{Cd}(\mathrm{OA})_{2}$ is injected to induce the reaction. Route 4: Cd(OA) $)_{2}$ in $\mathrm{ODE}$ solution is prepared and then the TOP-Se is injected. After $32 \mathrm{~s}$, OAm is injected into this reaction. X represents no reaction; $\{\mathrm{CdSe}\}$ represents the CdSe molecular building blocks ("monomers"), prepared without amine; $\{\mathrm{CdSe}\}_{\mathrm{OAm}}$ represents the CdSe monomers surrounded by OAm molecules; $\{\mathrm{CdSe}$ NCs $\}$ represents finally formed CdSe NCs without use of OAm, and $\{\mathrm{CdSe} \text { NCs }\}_{\mathrm{OAm}}$ represents finally formed CdSe NCs surrounded by OAm molecules.

of ODE, degassed for more than $20 \mathrm{~min}$, and stirred under $210^{\circ} \mathrm{C}$ for more than $10 \mathrm{~min} .0 .5 \mathrm{~mL}$ of Se precursor was then quickly injected, and timing started right at the moment. Aliquot volumes of the solution were fetched at different time interval for further analysis. In route 2, a certain amount of OAm was added with $\mathrm{Cd}$ precursor and kept stirring for $10 \mathrm{~min}$ at $210^{\circ} \mathrm{C}$ before the Se precursor injection. In contrast, in route 3, OAm was first mixed with Se precursor before the Cd precursor injection. In route 4, cold Se precursor was first injected into hot $\mathrm{Cd}$ precursor, and $32 \mathrm{~s}$ later, OAm was injected. The first aliquot sample was extracted before the amine injection. In all the amine-containing reaction, the amine contents include 0.1, 0.5, 1.0, and $2.5 \mathrm{mmol}$. Specially, for the amine injection solutions of $0.1,0.5$, and $1.0 \mathrm{mmol}$ in route 4 , a certain amount of ODE was added to keep them same volume as that of $2.5 \mathrm{mmol}$ in the case of the influence of different volumes.

Characterization. $50 \mu \mathrm{L}$ of as-synthesized CdSe NCs in ODE was diluted in $1450 \mu \mathrm{L}$ of hexane for the UV and fluorescence measurement without any purification. UV-vis absorbance spectroscopy was performed on a Varian Cary 100 Scan UV-vis spectrophotometer. Fluorescence spectra were measured by a twofiber system attached with a USB2000 spectrometer (Ocean Optics). The CdSe NCs were purified by repeated centrifugation and redispersion in a mixture of chloroform and ethanol (1:2) for 4 times and finally dissolved in hexane. TEM graphs and HRTEM graphs were captured by a Talos transmission electron microscope (FEI Co.), before which the CdSe NCs in hexane were dropped and dried in ultrathin carbon-supported copper grids.

NC Size, Growth Rate, and Concentration Calculation. The average sizes $(D)$ of the CdSe NCs were calculated based on the absorbance peak $(\lambda)$ of the as-prepared products and the empirical fitting function reported by Peng et al., ${ }^{21}$ given as

$$
\begin{aligned}
D & =(1.6122 \times 10-9) \times \lambda^{4}-(2.6575 \times 10-6) \times \lambda^{3} \\
& +(1.6242 \times 10-3) \times \lambda^{2}-(0.4277) \times \lambda+(41.57)
\end{aligned}
$$

The corresponding growth rates were obtained by the derivative of nanocrystal volumes with respect to time, that is

$$
R_{\text {Growth }}=\frac{\mathrm{d} V}{\mathrm{~d} t}=\frac{\pi}{6} \frac{\mathrm{d} D^{3}}{\mathrm{~d} t}
$$

The concentrations of the resulting $\mathrm{CdSe}$ NCs were estimated according to the method proposed by Bawendi et al. ${ }^{22}$ through counting the extinction coefficient at the wavelength of $350 \mathrm{~nm}$

$$
[\mathrm{NCs}]=\frac{A_{350}}{\varepsilon L}=\frac{8 A_{350}}{1.438 \times 10^{26} D^{3}}
$$

where $A_{350}, \varepsilon, L$, and $D$ are the absorbance intensity at the wavelength of $350 \mathrm{~nm}$, extinction coefficient per mole of NCs $\left(\mathrm{M}^{-1} \mathrm{~cm}^{-1}\right)$, path length of the radiation beam $(\mathrm{cm})$, and the corresponding average size of the CdSe NCs $(\mathrm{cm})$, respectively.

To calculate the total CdSe concentration in the form of NC, we can combine the known NC number and size by the following equation:

$$
[\mathrm{CdSe}]=N[\mathrm{NCs}]
$$

in which $N$ is the number of CdSe units in each particle. If we assume all the CdSe NC have a spherical shape and the solution has an extremely monodisperse population, then we can calculate $N$ as

$$
N=\frac{\pi}{6} D^{3} \frac{2}{V_{\text {unit }}}
$$

where $V_{\text {unit }}$ is the volume of the unit cell containing two CdSe units, which is equal to $0.112 \mathrm{~nm}^{3}$ in the case of the bulk wurtzite CdSe materials. ${ }^{22}$ The corresponding rate of CdSe formation (Figure S3) and the rate of nanocrystal number (Figure S4) were obtained by the derivative of the CdSe concentration and NCs concentration, respectively.

\section{RESULTS AND DISCUSSION}

To interpret the influence of OAm on the nucleation and growth behavior of NCs, different synthetic NCs routes were studied, by altering the time and sequence of addition of various reactants. Four reaction routes were designed, as schematically presented in Figure 1 . Route 1 (classical aminefree route): Cd oleate precursor $\left(\mathrm{Cd}(\mathrm{OA})_{2}\right)$ in octadecene (ODE) solution is prepared, and thereafter the Se precursor trioctylphosphine-selenium (TOP-Se) is injected. Route 2: various concentrations of $\mathrm{OAm}$ are added to the $\mathrm{Cd}(\mathrm{OA})_{2}$ in 
ODE solution, after which Se precursor is injected. Route 3: various concentrations of $\mathrm{OAm}$ and Se precursor are mixed together in $\mathrm{ODE}$, after which $\mathrm{Cd}(\mathrm{OA})_{2}$ is injected to induce the reaction. Route 4: $\mathrm{Cd}(\mathrm{OA})_{2}$ in ODE solution is prepared, and then the Se precursor (TOP-Se) is injected. After $32 \mathrm{~s}$, OAm is injected into this reaction mixture.

In route 2 , synthesis starts by reacting OAm with the cadmium precursor, followed by the addition of the selenium precursor. Accordingly, the influence of OAm starts already prior to the nucleation process, resulting in the difficulty of distinguishing the respective influence on nucleation and NC growth stage. Both routes 2 and 3 divulge the amine adduct formation with the cadmium precursor prior to the nucleation stage, whereas route 4 concentrates on the OAm influence on the NC growth stage, avoiding its influence in both initial precursor and nucleation stages. We benchmarked all results with respect to route 1 , which is the standard synthesis process. The reaction products from all the four routes were extracted at different times during the reaction to perform UV-vis absorbance and PL measurements. Figure 2 shows UV-vis
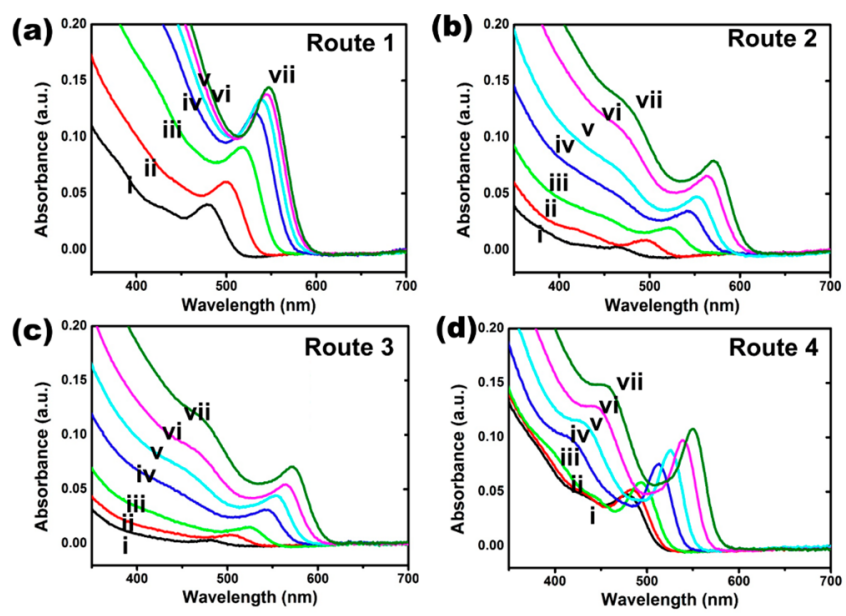

Figure 2. UV-vis absorbance spectra of CdSe NCs prepared using different routes: (a) route 1 in which no OAm was added; (b) route 2 in which TOP-Se was injected into a mixture of $\mathrm{Cd}(\mathrm{OA})_{2}$ and OAm; (c) route 3 in which $\mathrm{Cd}(\mathrm{OA})_{2}$ was injected into a mixture of TOP-Se and OAm; (d) route 4 in which TOP-Se was injected into $\mathrm{Cd}(\mathrm{OA})_{2}$, followed by the injection of OAm. Curves i, ii, iii, iv, v, vi, and vii in each graph are spectra of the reaction products extracted at a reaction time of $0.5,1,2,4,6,10$, and $15 \mathrm{~min}$, respectively. OAm amount used in reaction routes $2-4$ is $2.5 \mathrm{mmol}$.

absorbance spectra obtained for the four different routes (routes 1-4). Reactions of routes 2-4 were done in the presence of $2.5 \mathrm{mmol}$ of OAm. Curves i, ii, iii, iv, v, vi, and vii in each graph correspond to $\mathrm{CdSe}$ NCs extracted after a reaction time of $0.5,1,2,4,6,10$, and $15 \mathrm{~min}$, respectively. Figures $2 \mathrm{a}-\mathrm{d}$ are the results of NCs obtained from routes 1-4, respectively. It is evident that all the first excitonic peaks in the four reaction routes present red-shifts with time evolution, signifying a timedependent growth behavior of the NCs. Absorbance spectra of route 1 (Figure 2a) and route 4 (Figure 2d) look similar, having higher intensity (Figure S1a) and sharper peaks (Figure S1b) of the first excitonic peaks than those in route 2 (Figure $2 b$ ) and route 3 (Figure $2 \mathrm{c}$ ). This implies a difference in growth behavior due to the presence of OAm at the beginning of the reaction. In order to obtain comprehensive knowledge of the OAm influence, the reaction was carried out under various concentrations of OAm, namely $0.1 \mathrm{mmol}$ (i.e., a molar ratio of 1:2 between Cd precursor and OAm), $0.5 \mathrm{mmol}$ (1:10), 1 mmol (1:20), and $2.5 \mathrm{mmol}(1: 50)$.

Figure 3 shows the temporal evolution of the NCs size (a, d, $\mathrm{g})$, the volumetric growth rate $(\mathrm{b}, \mathrm{e}, \mathrm{h})$, and concentration $(\mathrm{c}, \mathrm{f}$, i) of CdSe NCs, prepared by route $2(a, b, c)$, route $3(d, e, f)$, and route $4(\mathrm{~g}, \mathrm{~h}, \mathrm{i})$, respectively. In general, the $\mathrm{CdSe}$ NCs size increases with time, and a higher OAm concentration results in a larger size. Also, a higher growth rate is obtained for higher OAm concentrations. Moreover, the time dependence of the NC concentration shows saturation for the longer reaction times with higher OAm concentrations resulting in lower concentrations. We also find results obtained using route 2 and 3 are comparable. At low OAm concentration $(<0.5 \mathrm{mmol})$, both routes show a smaller final NC size, lower initial growth rate, and higher final $\mathrm{NC}$ concentration compared to the standard route 1 (Figure S2). When amine content was above 1 mmol, however, an opposite result was obtained, namely, a larger final NC size, higher initial growth rate, and lower final NC concentration than obtained using route 1 . Most distinct information is the smaller initial size in route 2 with respect to route 3 . The OAm influence at the growth stage was examined using route 4 , in which OAm was injected $32 \mathrm{~s}$ after the reaction of $\mathrm{Cd}$ and Se precursors. At the time of injecting OAm, nucleation has already started (around 20 out of $30 \mu \mathrm{M}$ CdSe nuclei were already formed at $32 \mathrm{~s}$ ), and NCs of about $2.2 \mathrm{~nm}$ were formed and continued to grow (Figure $3 \mathrm{~g}$ ). It was seen that the final NC concentration was almost the same as with route 1, i.e., it was around $30 \mu \mathrm{M}$ (Figure S2c and Figure 3i), while in the case that $\mathrm{OAm}$ was fully mixed with $\mathrm{Cd}(\mathrm{OA})_{2}$ before the reaction (route 2), the NC concentration increased from $\sim 10$ to $42 \mu \mathrm{M}$ (Figure $3 \mathrm{c}$ ). This means the promotion of the NC nucleation at lower amine concentration (like 0.5 $\mathrm{mmol}$ ) is closely related to the injection of OAm. We also observed a sharp decline of the growth rate in the first minutes after injection of OAm. At lower OAm concentration, the growth rate is smaller but, for longer reaction times, similar to the other routes (Figure $3 \mathrm{~h}$ ). Therefore, NCs size in the final stage is smallest for route 4 . Growth rate evolution curves transform from an exponential-like decrease to a linear-like increase as a function of OAm concentration, which is clearly distinct from routes 2 and 3 .

Furthermore, we calculate the temporal evolution of the CdSe concentration in the form of NC, as shown in Figure 4. In general, the conversion of the precursor to CdSe NCs, no matter in which injection route, displays a decline with the increase of OAm amount. From routes 2 and 3, we can see that the addition of a little amount of OAm (like $0.5 \mathrm{mmol}$, or a molar ratio of 1:2 between $\mathrm{Cd}$ and OAm) has little effect on the $\mathrm{CdSe}$ formation. This is because the nucleation-promoting effect compensates for the reduction on the growth of the NCs. The nucleation-enhanced effect is weak in route 4, therefore resulting in an obvious reduced number of $\mathrm{CdSe}$ units even when only $0.5 \mathrm{mmol}$ OAm was injected (Figure $4 \mathrm{~d}$ ). At higher OAm concentration (above $1.0 \mathrm{mmol}$ ), although an enhancement on the NC growth rate is observed, the decreased production of new nuclei leads to a substantial reduction of the conversion to CdSe NCs. We also find the rate of CdSe formation (Figure S3) and the rate of nanocrystal number (Figure S4) have very similar trends with the increase of OAm concentration. These observations imply that for the conversion of precursors to CdSe units, the concentration of NC plays the dominant role rather than the size of the NC. At 

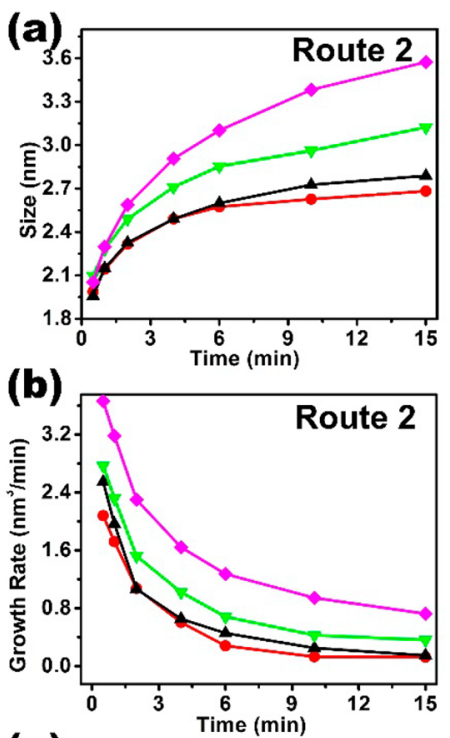

(c)

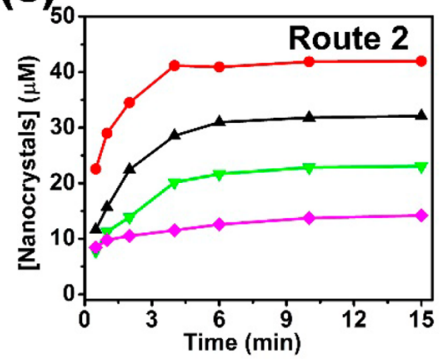

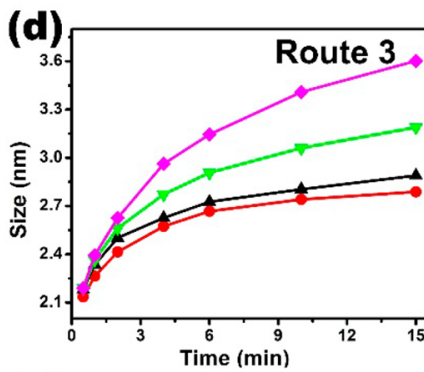

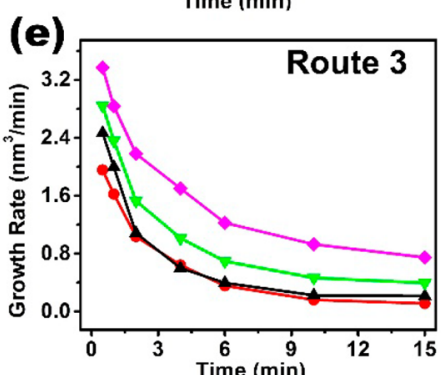

(f)

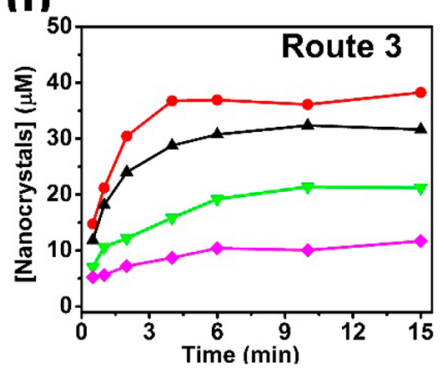

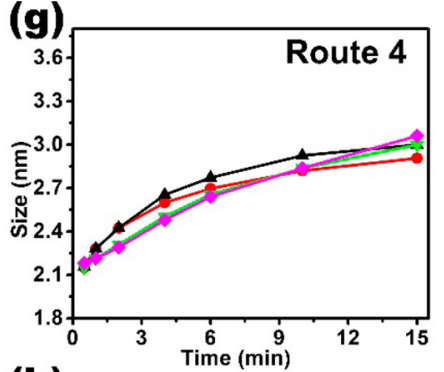

(h)

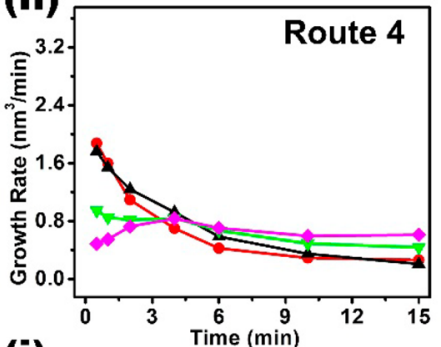

(i)

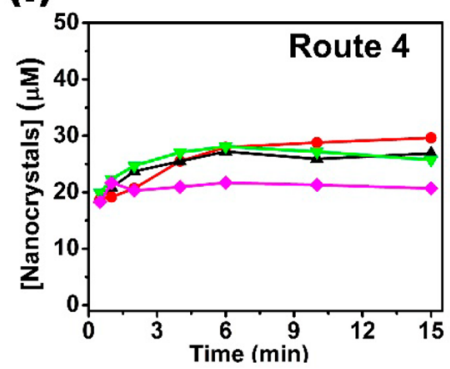

Figure 3. Temporal evolution of the size ( $a, d, g)$, volumetric growth rate (b, e, h), and NC concentration (c, f, i) of CdSe NCs prepared using route $2(\mathrm{a}, \mathrm{b}, \mathrm{c})$, in which TOP-Se was injected into a mixture of $\mathrm{Cd}(\mathrm{OA})_{2}$ and $\mathrm{OAm}$, using route $3(\mathrm{~d}, \mathrm{e}, \mathrm{f})$, in which $\mathrm{Cd}(\mathrm{OA})_{2}$ was injected into a mixture of TOP-Se and OAm, and using route $4(\mathrm{~g}, \mathrm{~h}, \mathrm{i})$, in which TOP-Se is injected into $\mathrm{Cd}(\mathrm{OA})_{2}$, followed by the injection of OAm after $32 \mathrm{~s}$ of reaction. All these reaction routes employed various amounts of OAm, namely, 0.1 $(\mathbf{\bullet}), 0.5(\boldsymbol{\Delta}), 1.0(\boldsymbol{\nabla})$, and $2.5 \mathrm{mmol}(\boldsymbol{\nabla})$.
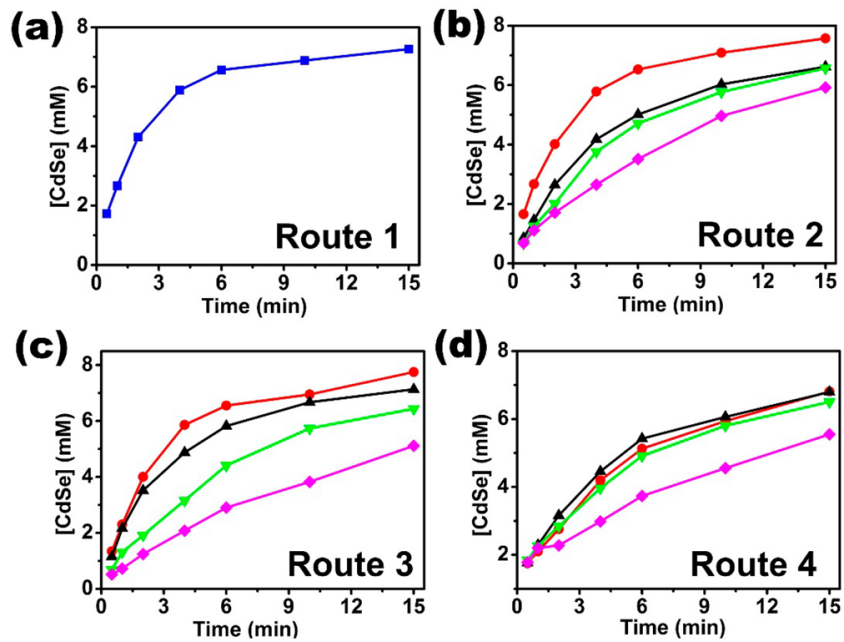

Figure 4. Temporal evolution of the CdSe concentration in the form of NC synthesized from route 1 (a) without amine addition, route 2 (b) in which TOP-Se was injected into a mixture of $\mathrm{Cd}(\mathrm{OA})_{2}$ and $\mathrm{OAm}$, route $3(\mathrm{c})$ in which $\mathrm{Cd}(\mathrm{OA})_{2}$ was injected into a mixture of TOP-Se and OAm, and route $4(\mathrm{~d})$ in which TOP-Se is injected into $\mathrm{Cd}(\mathrm{OA})_{2}$, followed by the injection of OAm after $32 \mathrm{~s}$ of reaction. All these reaction routes employed various amounts of OAm, namely, 0.1 $(\boldsymbol{\bullet}), 0.5(\boldsymbol{\Delta}), 1.0(\boldsymbol{\nabla})$, and $2.5 \mathrm{mmol}(\boldsymbol{\vee})$.

lower concentration, amine is mainly used to coordinate to the $\mathrm{Cd}$ center to form $\mathrm{Cd}$-amine complex precursor, thereby leading to the formation of high amounts of nuclei and CdSe units. At higher concentration, excess amine acts as the passivating agent, slowing down the nucleation process and thus resulting in reduced conversion of the precursors into CdSe products.

The full width at half-maximum (fwhm) of the PL is an indicator of size distribution of CdSe NCs. Figures 5a-c demonstrate fwhm of CdSe NCs obtained by (a) route 2, (b) route 3 , and (c) route 4 at a reaction time of $15 \mathrm{~min}$ with a OAm content of $2.5 \mathrm{mmol}$. Figures $5 \mathrm{~d}-\mathrm{f}$ show fwhm as a function of reaction times for routes 2,3 , and 4, respectively. During onset of NC growth, a rapid decline in fwhm during the first 2 min of the reaction happens, while for routes 2 and 3, a slow rise takes place at the later growth stage. Contrarily, route 4 shows a continuous fall of fwhm with time, suggesting the size focusing. Our results here demonstrate the impact of amine on the size distribution depends not only on the ligand amount but also on the way it is added. The increase of the amine amount is related to enhancement of the passivating effect, leading to the decrease of the NCs numbers and increase of the monomer supply for each NC during the growth stage. According to the size-focusing theory, when there are enough monomers in the solution, smaller particles can grow faster than the larger ones, eventually resulting in the size focusing. This is why we can see the fwhm decline with the increase of amine concentration in routes 2,3 , and 4 . Otherwise, in routes 2 and 3, the emergence of a gradual nucleation stage of around $4 \mathrm{~min}$ leads to an increase of the PL width of the NCs with time, while that has 

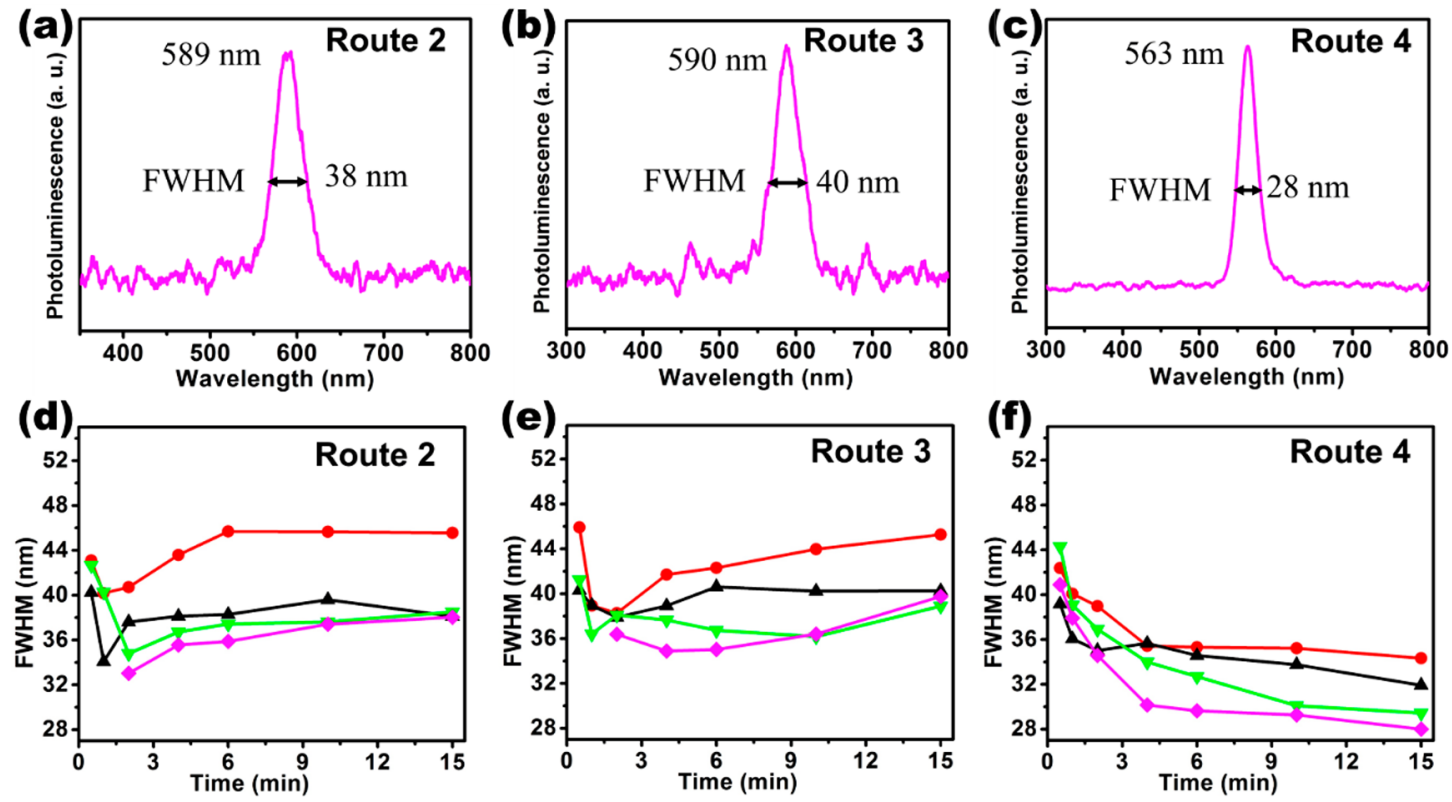

Figure 5. Photoluminescence (PL) spectra of CdSe NCs extracted after 15 min of reaction(a) using route 2, in which TOP-Se was injected into a mixture of $\mathrm{Cd}(\mathrm{OA})_{2}$ and $\mathrm{OAm},(\mathrm{b})$ using route 3 , in which $\mathrm{Cd}(\mathrm{OA})_{2}$ was injected into a mixture of TOP-Se and OAm, and (c) using route 4, in which TOP-Se was injected into $\mathrm{Cd}(\mathrm{OA})_{2}$, followed by the injection of OAm. Temporal evolution of fwhm of the PL peaks of CdSe NCs obtained using $(\mathrm{d})$ route $2,(\mathrm{e})$ route 3 , and (f) route 4 . All these reaction routes employed various amounts of OAm, namely, $0.1(\boldsymbol{\bullet}), 0.5(\boldsymbol{\Delta}), 1.0(\boldsymbol{\nabla})$, and $2.5 \mathrm{mmol}(\diamond)$.

been minimized in route 4 , resulting in a continuous decrease of the fwhm and better focusing of the NC size.

High-resolution transmission electron microscopy (HRTEM) graphs apprise the size, shape, size distribution, and crystal structure of CdSe NCs. Figure 6 shows TEM graphs (ad) and size distribution profiles $(\mathrm{e}-\mathrm{h})$ of the CdSe NCs obtained from route $1(\mathrm{a}, \mathrm{e})$, route $2(\mathrm{~b}, \mathrm{f})$, route $3(\mathrm{c}, \mathrm{g})$, and route $4(\mathrm{~d}, \mathrm{~h})$. The OAm amount used in routes $2-4$ is 0.5 mmol. The insets in $(\mathrm{a}-\mathrm{d})$ are the corresponding HRTEM graphs with lattice fringes. CdSe NCs obtained from all the routes possess a well-defined spherical shape. The average size of NCs based on the TEM graphs are 2.98, 2.83, 2.85, and 3.04 $\mathrm{nm}$ for routes $1-4$, respectively, well corresponding to the 3.00 , $2.79,2.89$, and $3.00 \mathrm{~nm}$ as calculated from the UV-vis absorbance data (Figure S1a and Figure $3 a-c)$. At higher OAm concentration like $2.5 \mathrm{mmol}$, the average NC sizes calculated from TEM are 3.59, 3.62, and $3.12 \mathrm{~nm}$ for routes 2-4, respectively (Figure S5), which is also in good agreement with those obtained from the absorbance spectra (3.60, 3.57, and $3.06 \mathrm{~nm}$ in Figures $3 \mathrm{a}-\mathrm{c}$, respectively). Very good crystallinity of these NCs is seen from the lattice fringe in HRTEM graphs. The calculated spacing is $0.36 \mathrm{~nm}$, which matches the (100) plane of a wurtzite CdSe NCs. ${ }^{23}$

All of these results suggest the following mechanism of OAm on the nucleation and growth processes of the NCs. In terms of precursor conversion, the addition of OAm would gradually decrease the formation of CdSe NCs, leading to about $30 \%$ loss of CdSe units the in the presence of $2.5 \mathrm{mmol}$ of OAm (Figure 4). However, with respect to the NC concentration and size, the tendency varies oppositely depending on the amount of $\mathrm{OAm}$. At lower OAm concentration (below $0.5 \mathrm{mmol}$ ), the NC number is increased while the corresponding NC size reduce. It is worth mentioning this phenomenon is different from the surface activation effect of alkylamine as reported by Peng et al. ${ }^{15-17}$ They claimed alkylamine would produce ZnSe NCs with smaller starting size, higher NC concentration, and higher growth rate, therefore contrary to the results we obtained here. They further proposed amine was responsible for accelerating the CdSe NCs growth when the amine was used above its boiling temperature (quasi-gas window), while in our case the reaction was performed at $210{ }^{\circ} \mathrm{C}$, far below the boiling temperature of $\mathrm{OAm}\left(364^{\circ} \mathrm{C}\right)$. We believe OAm here acts as a "nucleating agent", which was considered to strongly bind to the NC surface, forming insoluble surface precipitates and kinetically delaying both dissolution and growth of nuclei. ${ }^{24}$ Two observations are found to demonstrate the role of amine as nucleating agent. First, enhanced NC concentrations are found when the amine content is relatively low (below 0.5 mmol). Second, the dissolution of unstable nuclei, which happens in route 1 , disappears after the addition of alkylamine, indicating the unstable NC becomes more stable under the protection of amine.

The interaction of amine with precursors reduces the solubility of the monomer in the solution, forming a less stable monomer that is easier to precipitate and nucleate. We focus now on the temporal evolution of NC concentration for route 1, as shown in Figure S2c, and routes 2 and 3, as shown in Figure $3 c$,f. Standard route 1 , where amine is absent, shows a prolonged nucleation period of $4 \mathrm{~min}$, followed by partly dissolution of NCs. ${ }^{20-22}$ Critical NC radius dynamically grows larger at the cost of redissolution of unstable smaller NCs. In the presence of amine, the rapid increase of NC concentration is directly followed by a saturated plateau, and the final NC concentrations are increased (routes 2 and 3). Therefore, OAm addition is proved to be beneficial to the nucleation process by effectively protecting the formed NCs and greatly extending the nucleation period. As a consequence, the final NC size becomes smaller (Figure 3a,d), but the size uniformity becomes inferior (Figure 5d,e).

We found that application of a higher OAm concentration in routes 2 and 3 slowed down nucleation, as shown in Figure 3c,f. The increase of OAm content results in a decrease of the 

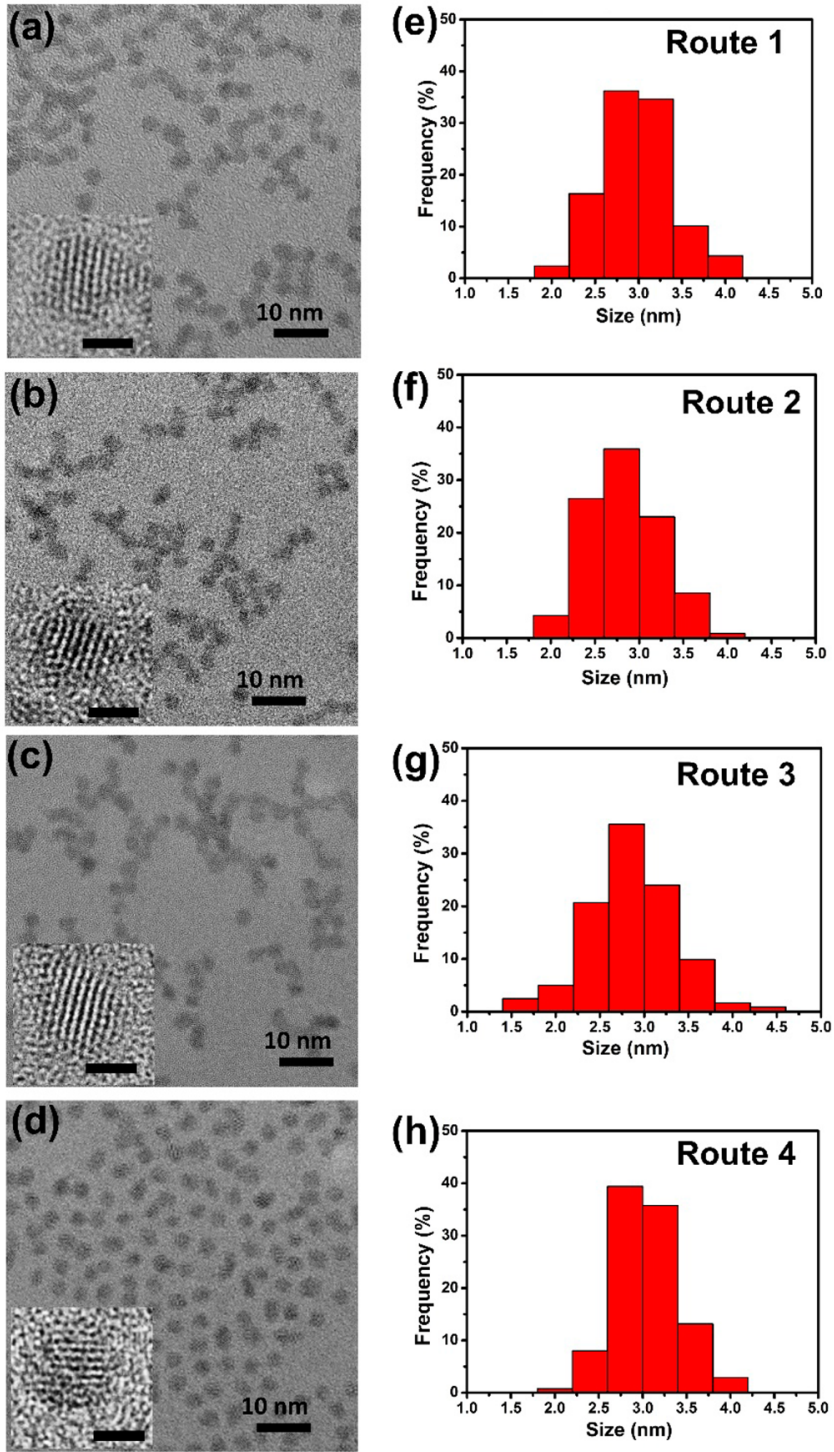

Figure 6. TEM graphs $(\mathrm{a}-\mathrm{d})$ and size distribution profiles $(\mathrm{e}-\mathrm{h})$ of the CdSe NCs. (a, e) CdSe NCs synthesized using route $1,(b, f) C d S e$ NCs synthesized using route $2,(c, g)$ CdSe NCs synthesized using route 3 , and $(\mathrm{d}, \mathrm{h}) \mathrm{CdSe} \mathrm{NCs}$ synthesized using route 4 . The OAm concentration in routes $2-4$ is $0.5 \mathrm{mmol}$. The insets in $(\mathrm{a}-\mathrm{d})$ are the corresponding HRTEM graphs with lattice fringes. Scale bars correspond to $2 \mathrm{~nm}$ for the insets.

collision frequency among nuclei, thereby suggesting OAm as a passivating agent. We know that the formation of nuclei comprised the binding of TOP-Se to the $\mathrm{Cd}$ center and cleavage of the $\mathrm{P}=\mathrm{Se}$ bond. In the absence of amine, cadmium oleate exists as a tetradentate complex. During the reaction, TOP-Se coordinates to the Cd center and forms an activated metal-phosphine complex at the expense of removing a carboxylate ion. Afterward, nucleophilic attack by the free carboxylate ion breaks the $\mathrm{P}=\mathrm{Se}$ bond and forms a $\mathrm{CdSe}$ bond (Figure 1a). ${ }^{27,28}$

When OAm is used prior to the nucleation, it would coordinate to the Cd center and form a hexadentate complex. Thereafter, this complex is bound to TOP-Se by substituting the amine ligand to form an alkylaminophosphonium intermediate, which is subsequently attacked by the carboxylate and finally reacts with $\mathrm{CdSe}$ monomer (Figure $1 \mathrm{~b}$ ). ${ }^{20}$
Compared to the tetradentate precursor, the hexadentate metal-amine complex would create stronger resistance for the binding of the $\mathrm{Cd}$ center with TOP-Se and thus deactivate the precursor reaction. Furthermore, excess amines are inclined to dynamically cover the nuclei, producing a steric hindrance, deterring the contact, collision, and agglomeration of the free monomers. This looks like an "isolation sphere" with nucleus as the core and OAm at the surface. Because of the excess of OAm, nucleation is impeded, subsequently resulting in higher $\mathrm{NC}$ growth rate. In route $4, \mathrm{OAm}$ was injected $32 \mathrm{~s}$ after the reaction of $\mathrm{Cd}$ and Se precursors, thus ensuring enough time for first nucleation to happen. However, subsequent nucleation slows down due to the addition of OAm, and this effect is more pronounced for the higher OAm concentrations. Further on in the process, growth rate and $\mathrm{NC}$ concentration have less dependence on OAm concentration, but the growth is slowed down with respect to the classical route 1 . This points to the main role of OAm as a passivating agent at the nucleation/ growth stage. In this way, an increase of the amine concentration would facilitate both the nucleation and growth.

\section{CONCLUSION}

We have established that OAm plays a dual role, acting on both nucleation and growth during synthesis of CdSe NCs by the hot injection method. The OAm concentration in the reaction mixture plays a key role. We found that at lower contents of OAm $(<0.5 \mathrm{mmol})$ it prominently acts as an agent affecting nucleation, thereby increasing the number of nuclei, increasing the NCs concentration, and thus decreasing the NCs size to 2.6 $\mathrm{nm}$, whereas at higher concentrations $(>1.0 \mathrm{mmol})$, it leads to passivation of nucleation as well as growth processes, so generating fewer NCs with larger size up to $3.7 \mathrm{~nm}$. Accordingly, adjusting degree of nucleation and passivation by OAm concentration, we can better control the final NCs size and so tune their size-dependent optical properties.

\section{ASSOCIATED CONTENT}

\section{Supporting Information}

The Supporting Information is available free of charge on the ACS Publications website at DOI: 10.1021/acs.langmuir.7b01337.

Results of classical CdSe synthetic route, including the temporal evolution of the NC size, growth rate, concentration, photoluminescence spectra, and full width at half-maximum (fwhm); the rate of CdSe concentration in the form of $\mathrm{NC}$ and rate of the $\mathrm{NC}$ number (PDF)

\section{AUTHOR INFORMATION}

\section{Corresponding Author}

*E-mail virendrakumar.parashar@epfl.ch (M.A.M.G.).

ORCID $\odot$

X. Huang: 0000-0002-4882-9331

V. K. Parashar: 0000-0003-2082-1028

\section{Notes}

The authors declare no competing financial interest.

\section{ACKNOWLEDGMENTS}

This work was financially supported by the Swiss National Science Foundation (Grant 200021-146237) and China Scholarship Council (No. 201406360047). 


\section{REFERENCES}

(1) Konstantatos, G.; Sargent, E. H. Colloidal Quantum Dot Optoelectronics and Photovoltaics; Cambridge University Press: New York, 2013.

(2) Bera, D.; Qian, L.; Tseng, T. K.; Holloway, P. H. Quantum dots and their multimodal applications: a review. Materials 2010, 3 (4), $2260-2345$.

(3) Green, M. Semiconductor Quantum Dots:Organometallic and Inorganic Synthesis; Royal Society of Chemistry: Cambridge, UK, 2014.

(4) Murray, C.; Norris, D. J.; Bawendi, M. G. Synthesis and characterization of nearly monodisperse $\mathrm{CdE}(\mathrm{E}=$ sulfur, selenium, tellurium) semiconductor nanocrystallites. J. Am. Chem. Soc. 1993, 115 (19), 8706-8715.

(5) Peng, Z. A.; Peng, X. Formation of high-quality CdTe, CdSe, and CdS nanocrystals using CdO as precursor. J. Am. Chem. Soc. 2001, 123 (1), 183-184.

(6) Peng, X.; Manna, L.; Yang, W.; Wickham, J.; Scher, E.; Kadavanich, A.; Alivisatos, A. P. Shape control of CdSe nanocrystals. Nature 2000, 404 (6773), 59-61.

(7) Donegá, C. M. Synthesis and properties of colloidal heteronanocrystals. Chem. Soc. Rev. 2011, 40 (3), 1512-1546.

(8) Grandhi, G. K.; M, A.; Viswanatha, R. Understanding the role of surface capping ligands in passivating the quantum dots using copper dopants as internal sensor. J. Phys. Chem. C 2016, 120 (35), 1978519795.

(9) Ko, J. H.; Yoo, D.; Kim, Y. H. Atomic models for anionic ligand passivation of cation-rich surfaces of IV-VI, II-VI, and III-V colloidal quantum dots. Chem. Commun. 2017, 53, 388-391.

(10) Talapin, D. V.; Rogach, A. L.; Kornowski, A.; Haase, M.; Weller, $\mathrm{H}$. Highly luminescent monodisperse $\mathrm{CdSe}$ and $\mathrm{CdSe} / \mathrm{ZnS}$ nanocrystals synthesized in a hexadecylamine-trioctylphosphine oxidetrioctylphospine mixture. Nano Lett. 2001, 1 (4), 207-211.

(11) Talapin, D. V.; Rogach, A. L.; Mekis, I.; Haubold, S.; Kornowski, A.; Haase, M.; Weller, H. Synthesis and surface modification of aminostabilized CdSe, CdTe and InP nanocrystals. Colloids Surf., A 2002, 202 (2), 145-154.

(12) Huang, X.; Parashar, V. K.; Gijs, M. A. M. Synergistic effect of carboxylic and amine ligands on the synthesis of CdSe nanocrystals. RSC Adv. 2016, 6 (91), 88911-88915.

(13) Boles, M. A.; Ling, D.; Hyeon, T.; Talapin, D. V. The surface science of nanocrystals. Nat. Mater. 2016, 15, 141-153.

(14) Drijvers, E.; De Roo, J.; Martins, J. C.; Infante, I.; Hens, Z. Ligand displacement exposes binding site heterogeneity on $\mathrm{CdSe}$ nanocrystal surfaces. Chem. Mater. 2018, 30 (3), 1178-1186.

(15) Li, L. S.; Pradhan, N.; Wang, Y.; Peng, X. High quality ZnSe and $\mathrm{ZnS}$ nanocrystals formed by activating zinc carboxylate precursors. Nano Lett. 2004, 4 (11), 2261-2264.

(16) Pradhan, N.; Reifsnyder, D.; Xie, R.; Aldana, J.; Peng, X. Surface ligand dynamics in growth of nanocrystals. J. Am. Chem. Soc. 2007, 129 (30), 9500-9509.

(17) Sun, Z.; Oyanagi, H.; Nakamura, H.; Jiang, Y.; Zhang, L.; Uehara, M.; Yamashita, K.; Fukano, A.; Maeda, H. Ligand effects of amine on the initial nucleation and growth processes of $\mathrm{CdSe}$ nanocrystals. J. Phys. Chem. C 2010, 114 (22), 10126-10131.

(18) Foos, E. E.; Wilkinson, J.; Mäkinen, A. J.; Watkins, N. J.; Kafafi, Z. H.; Long, J. P. Synthesis and surface composition study of CdSe nanoclusters prepared using solvent systems containing primary, secondary, and tertiary amines. Chem. Mater. 2006, 18 (12), 28862894.

(19) Jose, R.; Zhanpeisov, N. U.; Fukumura, H.; Baba, Y.; Ishikawa, M. Structure-property correlation of CdSe clusters using experimental results and first-principles DFT calculations. J. Am. Chem. Soc. 2006, 128 (2), 629-636.

(20) García-Rodríguez, R.; Liu, H. Mechanistic insights into the role of alkylamine in the synthesis of CdSe nanocrystals. J. Am. Chem. Soc. 2014, 136 (5), 1968-1975.

(21) Yu, W. W.; Qu, L.; Guo, W.; Peng, X. Experimental determination of the extinction coefficient of CdTe, CdSe, and CdS Nanocrystals. Chem. Mater. 2003, 15 (14), 2854-2860.
(22) Leatherdale, C. A.; Woo, W.-K.; Mikulec, F. V.; Bawendi, M. G. On the Absorption Cross Section of CdSe Nanocrystal Quantum Dots. J. Phys. Chem. B 2002, 106 (31), 7619-7622.

(23) Chen, Y.; Wei, L.; Zhang, G.; Jiao, J. Open structure ZnO/CdSe core/shell nanoneedle arrays for solar cells. Nanoscale Res. Lett. 2012, 7 (1), 516-521.

(24) Van Embden, J.; Mulvaney, P. Nucleation and growth of CdSe nanocrystals in a binary ligand system. Langmuir 2005, 21 (22), 10226-10233.

(25) Owen, J. S.; Chan, E. M.; Liu, H.; Alivisatos, A. P. Precursor conversion kinetics and the nucleation of cadmium selenide nanocrystals. J. Am. Chem. Soc. 2010, 132 (51), 18206-18213.

(26) Bullen, C. R.; Mulvaney, P. Nucleation and growth kinetics of CdSe nanocrystals in octadecene. Nano Lett. 2004, 4 (12), 23032307.

(27) Liu, H.; Owen, J. S.; Alivisatos, A. P. Mechanistic study of precursor evolution in colloidal group II-VI semiconductor nanocrystal synthesis. J. Am. Chem. Soc. 2007, 129 (2), 305-312.

(28) García-Rodríguez, R. Liu, H. Mechanistic Study of the Synthesis of CdSe Nanocrystals: Release of Selenium. J. Am. Chem. Soc. 2012, 134 (3), 1400-1403. 